\title{
Direct conversion of biomass carbohydrates to platform chemicals: 5-hydroxymethylfurfural (HMF) and furfural
}

\author{
Ashutosh Mittal ${ }^{*}$, Heidi M. Pilath, David K. Johnson \\ Biosciences Center, National Renewable Energy Laboratory, Golden, Colorado 80401 \\ *Email_Ashutosh.Mittal@nrel.gov
}

Mailing address: Biosciences Center,

National Renewable Energy Laboratory

15013 Denver West Parkway

Golden, CO 80401-3305, USA

Number of pages: 4

Number of Figures: 4

Number of Tables: 2 


\section{Supplemental Materials - Figures and Tables}

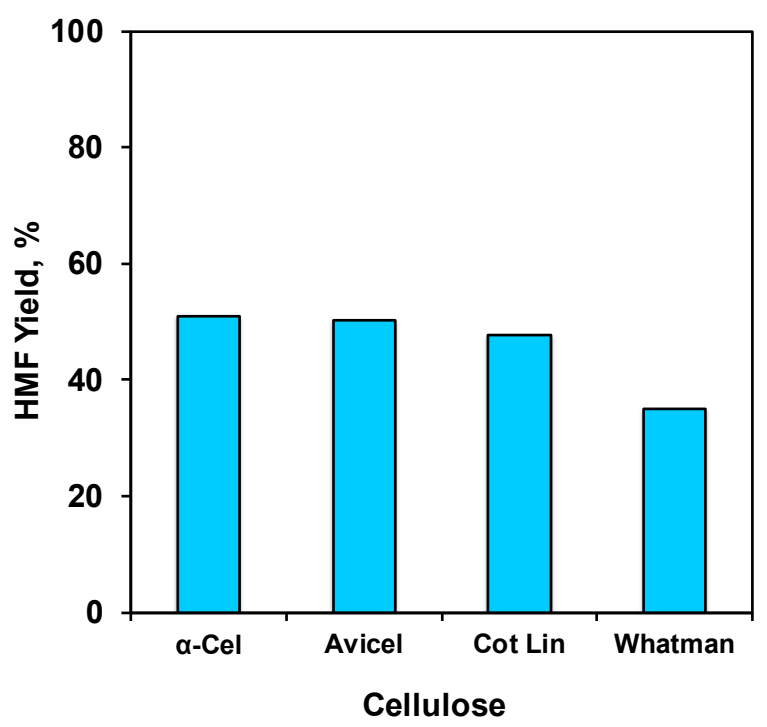

Figure S1. HMF yields from dehydration of various commercial celluloses, $\alpha$-cellulose $(\alpha$-Cel), Avicel PH101 (Avicel), cotton linters (Cot Lin), and Whatman filter paper No. 1 (Whatman) using $33 \mathrm{mM} \mathrm{HCl}$ and 8 $\mathrm{mM} \mathrm{AlCl}{ }_{3}$ at $200^{\circ} \mathrm{C}$ for $5 \mathrm{~min}$. Dioxane to aqueous ratio was 2:1.

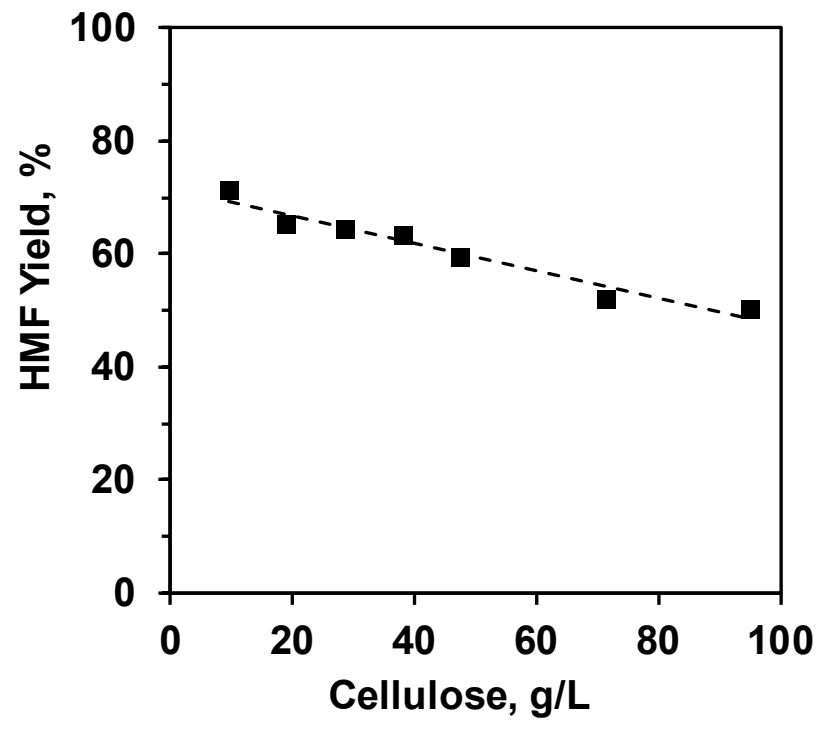

Figure S2. HMF yield from dehydration of Avicel PH 101 at different cellulose loadings using $33 \mathrm{mM} \mathrm{HCl}$ and $8 \mathrm{mM} \mathrm{AlCl}_{3}$ at $200^{\circ} \mathrm{C}$ for $5 \mathrm{~min}$. Dioxane to aqueous ratio was 3:1. 


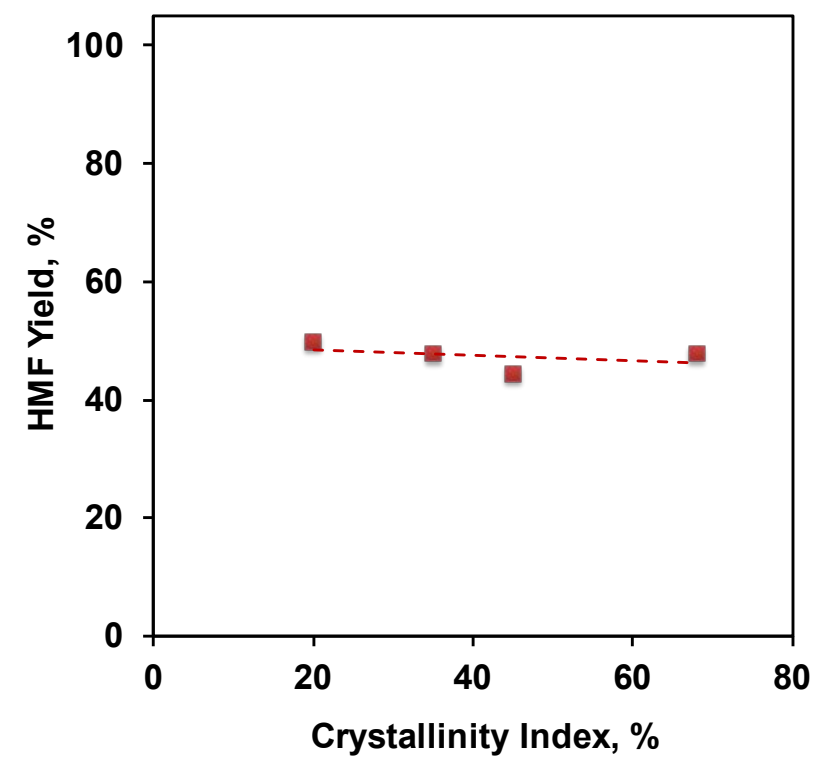

Figure S3. HMF yield from dehydration of cotton linters as a function of crystallinity index using $33 \mathrm{mM}$ $\mathrm{HCl}$ and $8 \mathrm{mM} \mathrm{AlCl}_{3}$ at $200^{\circ} \mathrm{C}$ for $5 \mathrm{~min}$. Dioxane to aqueous ratio was 2:1.

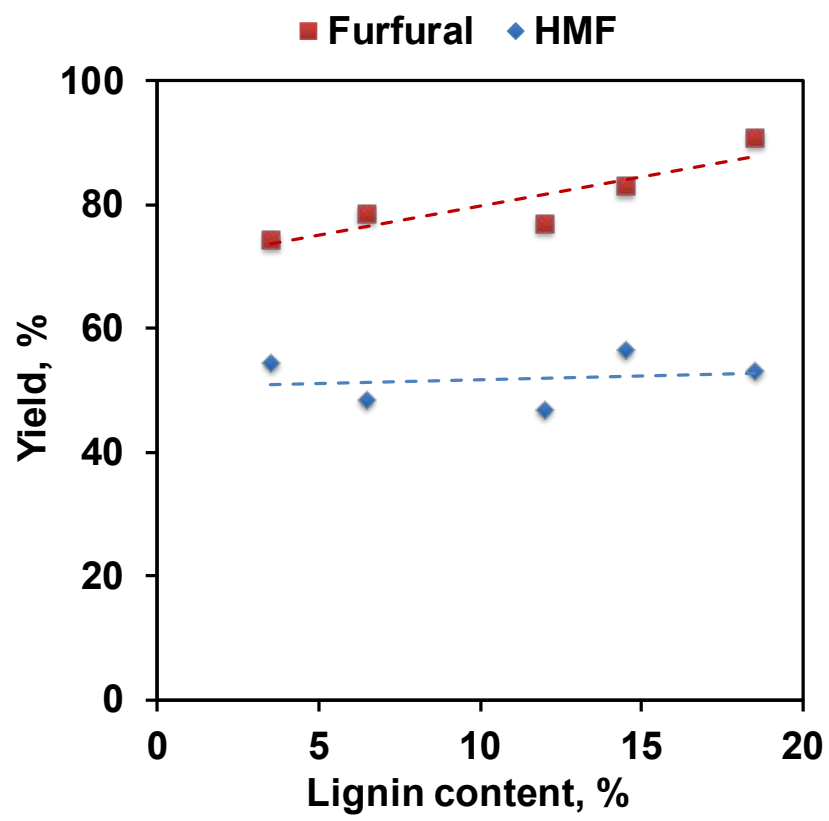

Figure S4. Furfural and HMF yields from dehydration of hydrogen peroxide treated corn stover at different lignin contents to evaluate the effect of lignin on furfurals production using $33 \mathrm{mM} \mathrm{HCl}$ and $8 \mathrm{mM} \mathrm{AlCl}_{3}$ at $200^{\circ} \mathrm{C}$ for $5 \mathrm{~min}$. Dioxane to aqueous ratio was 2:1 
Table S1. Glucan contents and Crl of various cellulose samples. CL1-CL3 are low crystallinity cellulose samples prepared from cotton linters.

\begin{tabular}{ccc}
\hline Feedstock & Glucan & Crl \\
\hline$\alpha$-Cellulose & 92.3 & 59.0 \\
Avicel PH 101 & 95.4 & 69.2 \\
Cotton linters & 99.1 & $68.0 \pm 1.3$ \\
Whatman No. 1 & 99.0 & 65.9 \\
CL-1 & $>99.0$ & 17.0 \\
CL-2 & $>99.0$ & 35.0 \\
CL-3 & $>99.0$ & 45.0 \\
\hline
\end{tabular}

Table S2. Compositions of $\mathrm{H}_{2} \mathrm{O}_{2}$ pretreated corn stover.

\begin{tabular}{ccccccc}
\hline $\begin{array}{c}\mathrm{H}_{2} \mathrm{O}_{2} \text { loading, } \\
\text { mg/g CS }\end{array}$ & $\begin{array}{c}\text { Solid } \\
\text { yield, } \%\end{array}$ & Glucan & Xylan & Arabinan & Acetate & Lignin \\
\hline $\begin{array}{c}\text { Untreated } \\
\text { Corn Stover }\end{array}$ & & $36.3 \pm 2.3$ & $26.4 \pm 2.2$ & $5.3 \pm 1.4$ & $2.5 \pm 0.7$ & $18.5 \pm 1.6$ \\
90 & 73.8 & 45.3 & 29.2 & 5.0 & - & 14.6 \\
125 & $71.9 \pm 1.5$ & $48.4 \pm 1.0$ & $29.4 \pm 1.0$ & $4.6 \pm 0.5$ & - & $11.9 \pm 1.6$ \\
250 & $57.7 \pm 2.6$ & $58.2 \pm 1.2$ & $30.1 \pm 0.3$ & $5.0 \pm 0.3$ & - & $6.5 \pm 1.5$ \\
500 & 51.0 & 65.2 & 27.1 & 4.3 & - & 3.5 \\
\hline
\end{tabular}

